of an outbreak of 2019 novel coronavirus diseases (COVID-19) - China, 2020. China CDC Weekly 2020 [cited 2020 Feb 29]. http:// weekly.chinacdc.cn/en/article/id/ e53946e2-c6c4-41e9-9a9b-fea8db1a8f51

Address for correspondence: Nick Wilson, Department of Public Health, University of Otago Wellington, Mein St, Newtown, Wellington 6005, New Zealand; email: nick.wilson@otago.ac.nz

\section{Serial Interval of COVID-19 among Publicly Reported Confirmed Cases}

\author{
Zhanwei Du, ${ }^{1}$ Xiaoke $\mathrm{Xu},{ }^{1}$ Ye Wu, ${ }^{1}$ Lin Wang, \\ Benjamin J. Cowling, Lauren Ancel Meyers \\ Author affiliations: University of Texas at Austin, Austin, \\ Texas, USA (Z. Du, L.A. Meyers); Dalian Minzu University, \\ Dalian, China (X. Xu); Beijing Normal University Computational \\ Communication Research Center, Zhuhai, China (Y. Wu); \\ Beijing Normal University School of Journalism and \\ Communication, Beijing, China (Y. Wu); Institut Pasteur, Paris, \\ France (L. Wang); University of Hong Kong, Hong Kong, \\ China (B.J. Cowling); Santa Fe Institute, Santa Fe, New Mexico, \\ USA (L.A. Meyers)
}

We estimate the distribution of serial intervals for 468 confirmed cases of coronavirus disease reported in China as of February 8, 2020. The mean interval was 3.96 days (95\% Cl 3.53-4.39 days), SD 4.75 days (95\% Cl 4.465.07 days); $12.6 \%$ of case reports indicated presymptomatic transmission.

DOI: https://doi.org/10.3201/eid2606.200357

K ey aspects of the transmission dynamics of coronavirus disease (COVID-19) remain unclear (1). The serial interval of COVID-19 is defined as the time duration between a primary case-patient (infector) having symptom onset and a secondary case-patient (infectee) having symptom onset (2). The distribution of COVID-19 serial intervals is a critical input for determining the basic reproduction number $\left(R_{0}\right)$

${ }^{1}$ These first authors contributed equally to this article. and the extent of interventions required to control an epidemic (3).

To obtain reliable estimates of the serial interval, we obtained data on 468 COVID-19 transmission events reported in mainland China outside of Hubei Province during January 21-February 8, 2020. Each report consists of a probable date of symptom onset for both the infector and infectee, as well as the probable locations of infection for both case-patients. The data include only confirmed cases compiled from online reports from 18 provincial centers for disease control and prevention (https://github.com/MeyersLabUTexas/COVID-19).

Fifty-nine of the 468 reports indicate that the infectee had symptoms earlier than the infector. Thus, presymptomatic transmission might be occurring. Given these negative-valued serial intervals, COVID-19 serial intervals seem to resemble a normal distribution more than the commonly assumed gamma or Weibull distributions $(4,5)$, which are limited to positive values (Appendix, https:/ / wwwnc.cdc.gov/EID/ article/26/7/20-0357-App1.pdf). We estimate a mean serial interval for COVID-19 of 3.96 (95\% CI 3.53-4.39) days, with an SD of 4.75 (95\% CI 4.46-5.07) days (Figure), which is considerably lower than reported mean serial intervals of 8.4 days for severe acute respiratory syndrome (5) to 14.6 days (6) for Middle East respiratory syndrome. The mean serial interval is slightly but not significantly longer when the index case is imported (4.06 [95\% CI 3.55-4.57] days) versus locally infected (3.66 [95\% CI 2.84-4.47] days), but slightly shorter when the secondary transmission occurs within the household (4.03 [95\% CI 3.12-4.94] days) versus outside the household (4.56 [95\% CI 3.85-5.27] days). Combining these findings with published estimates for the early exponential growth rate COVID-19 in Wuhan (7), we estimate an $\mathrm{R}_{0}$ of 1.32 (95\% CI 1.16-1.48) (5), which is lower than published estimates that assume a mean serial interval exceeding 7 days $(7,8)$.

These estimates reflect reported symptom onset dates for 752 case-patients from 93 cities in China, who range in age from 1 to 90 years (mean 45.2 years, SD 17.21 years). Recent analyses of putative COVID-19 infector-infectee pairs from several countries have indicated average serial intervals of 4.0 days (95\% CI 3.1-4.9 days; $n=28$; unpub. data, H. Nishiura et al., unpub. data, https:// doi.org/10.1101/2020.02. 03.20019497), 4.4 days (95\% CI 2.9-6.7 days, $\mathrm{n}=21$; $\mathrm{S}$. Zhao et al., unpub. data, https:// doi.org/10.1101/20 20.02.21.20026559], and 7.5 days (95\% CI 5.3-19, $\mathrm{n}=$ $6 ; 8)$. Whereas none of these studies report negative serial intervals in which the infectee had symptoms before the infector, $12.6 \%$ of the serial intervals in our sample were negative. 
Figure. Estimated serial interval distribution for coronavirus disease (COVID-19) based on 468 reported transmission events, China, January 21-February 8, 2020. A) All infection events $(\mathrm{N}=468)$ reported across 93 cities of mainland China as of February 8, 2020; B) the subset infection events $(\mathrm{n}=$ 122 ) in which both the infector and infectee were infected in the reporting city (i.e., the index patient's case was not an importation from another city). Gray bars indicate the number of infection events with specified serial interval, and blue lines indicate fitted normal distributions. Negative serial intervals (left of the vertical dotted lines) suggest the possibility of COVID-19 transmission from asymptomatic or mildly symptomatic case-patients.
We note 4 potential sources of bias. First, the data are restricted to online reports of confirmed cases and therefore might be biased toward more severe cases in areas with a high-functioning healthcare and public health infrastructure. The rapid isolation of such case-patients might have prevented longer serial intervals, potentially shifting our estimate downward compared with serial intervals that might be observed in an uncontrolled epidemic. Second, the distribution of serial intervals varies throughout an epidemic; the time between successive cases contracts around the epidemic peak (9). A susceptible person is likely to become infected more quickly if they are surrounded by 2 infected persons instead of 1 . Because our estimates are based primarily on transmission events reported during the early stages of outbreaks, we do not explicitly account for such compression and interpret the estimates as basic serial intervals at the outset of an epidemic. However, if some of the reported infections occurred amid growing clusters of cases, then our estimates might reflect effective (compressed) serial intervals that would be expected during a period of epidemic growth. Third, the identity of each infector and the timing of symptom onset were presumably based on individual recollection of past events. If recall accuracy is impeded by time or trauma, casepatients might be more likely to attribute infection to recent encounters (short serial intervals) over past encounters (longer serial intervals). In contrast, the reported serial intervals might be biased upward by travel-related delays in transmission from primary case-patients that were infected in Wuhan or another city before returning home. If their infectious period started during travel, then we might be unlikely to observe early transmission events with shorter serial intervals. The mean serial interval is slightly higher for the 218 of 301 unique infectors reported to have imported cases.

Given the heterogeneity in type and reliability of these sources, we caution that our findings should be interpreted as working hypotheses regarding the infectiousness of COVID-19, requiring further validation. The potential implications for COVID-19 control are mixed. Although our lower estimates for $\mathrm{R}_{0}$ suggest easier containment, the large number of reported asymptomatic transmission events is concerning.

We acknowledge the financial support from the US National Institutes of Health (grant no. U01 GM087719) and the National Natural Science Foundation of China (grant no. 61773091).

\section{About the Author}

Dr. Du is a postdoctoral researcher in the Department of Integrative Biology at the University of Texas at Austin. He develops mathematical models to elucidate the transmission dynamics, surveillance, and control of infectious diseases.

\section{References}

1. Cowling BJ, Leung GM. Epidemiological research priorities for public health control of the ongoing global novel coronavirus (2019-nCoV) outbreak. Euro Surveill. 2020;25. https://doi.org/10.2807/1560-7917.ES.2020.25.6.2000110

2. Svensson A. A note on generation times in epidemic models. Math Biosci. 2007;208:300-11. https://doi.org/10.1016/ j.mbs.2006.10.010

3. Wallinga J, Lipsitch M. How generation intervals shape the relationship between growth rates and reproductive numbers. Proc Biol Sci. 2007;274:599-604. https:/ / doi.org/ 10.1098/rspb.2006.3754

4. Kuk AYC, Ma S. The estimation of SARS incubation distribution from serial interval data using a convolution likelihood. Stat Med. 2005;24:2525-37. https:/ / doi.org/10.1002/sim.2123

5. Lipsitch M, Cohen T, Cooper B, Robins JM, Ma S, James L, et al. Transmission dynamics and control of severe acute 
respiratory syndrome. Science. 2003;300:1966-70.

https://doi.org/10.1126/science.1086616

6. Park SH, Kim Y-S, Jung Y, Choi SY, Cho N-H, Jeong HW, et al. Outbreaks of Middle East respiratory syndrome in two hospitals initiated by a single patient in Daejeon, South Korea. Infect Chemother. 2016;48:99-107. https:/ / doi.org/ 10.3947/ic.2016.48.2.99

7. Li Q, Guan X, Wu P, Wang X, Zhou L, Tong Y, et al. Early transmission dynamics in Wuhan, China, of novel coronavirusinfected pneumonia. N Engl J Med. 2020 Jan 29 [Epub ahead of print]. https://doi.org/10.1056/NEJMoa2001316

8. Wu JT, Leung K, Leung GM. Nowcasting and forecasting the potential domestic and international spread of the 2019-nCoV outbreak originating in Wuhan, China: a modelling study. Lancet. 2020;395:689-97. https://doi.org/ 10.1016/S0140-6736(20)30260-9

9. Kenah E, Lipsitch M, Robins JM. Generation interval contraction and epidemic data analysis. Math Biosci. 2008;213:71-9. https:// doi.org/10.1016/j.mbs.2008.02.007

Address for correspondence: Lauren Ancel Meyers, J.T. Patterson Labs Bldg, University of Texas at Austin, 2415 Speedway, Austin, TX 78712, USA; email: laurenmeyers@austin.utexas.edu

\section{Indirect Virus Transmission in Cluster of COVID-19 Cases, Wenzhou, China, 2020}

\author{
Jing Cai, ${ }^{1}$ Wenjie Sun, ${ }^{1}$ Jianping Huang, ${ }^{1}$ \\ Michelle Gamber, Jing Wu, Guiqing $\mathrm{He}$
}

Author affiliations: Wenzhou Sixth People's Hospital, Wenzhou Central Hospital Medical Group, Wenzhou, China (J. Cai, J. Huang, G. He); The Second Affiliated Hospital of Fujian Traditional Chinese Medical University, Fuzhou, China (W. Sun); Robert Stempel College of Public Health and Social Work, Florida International University, Miami, Florida, USA (W. Sun); Shenandoah University, Winchester, Virginia, USA (M. Gamber); Huashan Hospital, Fudan University, Shanghai, China (J. Wu)

DOI: https://doi.org/10.3201/eid2606.200412

To determine possible modes of virus transmission, we investigated a cluster of coronavirus disease cases associated with a shopping mall in Wenzhou, China. Data indicated that indirect transmission of the causative virus occurred, perhaps resulting from virus contamination of common objects, virus aerosolization in a confined space, or spread from asymptomatic infected persons.

${ }^{1}$ These authors contributed equally to this article.
Severe acute respiratory syndrome coronavirus 2 S(SARS-CoV-2), the causative agent of coronavirus disease (COVID-19), is presumed to spread primarily via respiratory droplets and close contact. However, these transmission modes do not explain all cases. To determine how the virus may have spread among a cluster of COVID-19 cases associated with a shopping mall in Wenzhou (a city with 8 million residents), China, we monitored and traced close contacts and hypothesized possible transmission modes. We analyzed clinical and laboratory data for cases by using real-time reverse transcription PCR (1). The study was approved with written consent from the Ethics Committee of Wenzhou Central Hospital and written informed consent from all case-patients.

On January 20, 2020, a 23-year-old man (patient E) sought care at a hospital after 11 days of fever and headache. On January 21, COVID-19 was confirmed for patient $\mathrm{E}$ and his co-worker, patient $\mathrm{G}$. The Wenzhou Center for Disease Control and Prevention traced and tested their contacts, and by January 28 , COVID-19 was confirmed for 7 persons (patients A-G) from the same office (on floor 7).

Patient A, a 30-year-old woman, the only case-patient who indicated that she had been in Wuhan, China, returned from Wuhan on December 18, 2019. On January 15-16, 2020, she had a fever, but symptoms resolved without treatment. Despite symptom resolution, on January 30 she was confirmed to have SARS-CoV-2 infection. If patient $\mathrm{A}$ is the index patient, infected in Wuhan, her incubation period would have been 28 days, which would be extremely long, according to updated information (W.J. Guan et al., unpub. data, https://www. medrxiv.org/content/10.1101/2020.02.06.20020974v1). Asymptomatic carrier transmission has been reported for SARS-CoV-2 (2); hence, patient A could have been screened as a close contact during her incubation period and then hospitalized on the basis of a positive test (PCR) result only. However, her clinical symptoms did not appear until after hospitalization. Because persons with asymptomatic COVID-19 can spread the virus, patient $\mathrm{A}$ also could have been an asymptomatic carrier with a persistent infection (3).

On January 22, the mall was shut down. During January 19-February 9, COVID-19 was diagnosed for 7 mall staff from floors B1-3 and for 10 mall customers. Close contacts associated with the mall were traced, and COVID-19 was confirmed for 11 persons. Sixteen patients had had direct contact with other patients or had gone shopping in the mall. The average incubation period was 7.3 (range 1-17) days.

The mall has 8 floors above ground and several basement levels; floors B1 to 6 are commercial 